\title{
The Focused Ion Beam Hard Mask: Insights Into the Mechanism and a New A pplication for Photonic Structures
}

\author{
W.R. McKenzie*, M.P. Hiscocks**, F. Ladouceur** and P.R. Munroe* \\ * Electron Microscope Unit, University of New South Wales, Sydney NSW 2052, Australia \\ ** Photonics Group, Electrical Engineering and Telecommunications, University of New South \\ Wales, Sydney NSW 2052, Australia
}

The recent discovery of the Focused Ion Beam Hard Mask (FIBHM) method by McKenzie et al., (2009) [1-2] represents a significant leap forward in the ability to pattern nano-scaled structures onto diamond surfaces. It also represents a new form of focused ion beam (FIB) lithography, which does not require the deposition of a sacrificial layer, such as a resist. Given diamond's portfolio of compelling material properties this advance will potentially enable a number of emerging technologies, or otherwise will make processing steps in existing technologies significantly simpler. To date, the FIBHM has been predicted to outperform resist-based lithographic techniques in ultimate feature resolution [2] and has proven itself as an enabling means to pattern nano-imprint dies capable of re-producing sub-100nm features imprinted into silicon [1].

The FIBHM process is illustrated in the schematic shown in Fig. 1 and produces structures such as those shown in the SEM images (A) and (B). From the schematic in Fig. 1, Step 1 involves locally implanting the surface with a $30 \mathrm{kV} \mathrm{Ga}^{+} \mathrm{FIB}$ to a dose of at least $7.5 \times 10^{15} \mathrm{~cm}^{-2}$. Step 2 involves exposing the entire surface to a plasma created using a reactive ion or plasma etch system. The non-FIB patterned diamond is etched by the plasma while the $\mathrm{Ga}^{+}$implanted diamond from Step 1 creates a mask that locally prevents etching, the so-called FIBHM.

An interesting characteristic of both Fig. 1(A) and (B) is that they have both created near identical results to those using a Step 2 etch from an argon and an oxygen plasma etch (respectively). This observation suggests that: 1) the masking mechanism is identical for etching treatments using both plasmas, and 2) the mask is effective against physical sputtering; the only etching mechanism possible in an Ar plasma, as it is chemically inert. This result contradicts findings for other masking mechanisms applied to diamond, which describe the mask as a chemical barrier that suppresses the reactive processes responsible for the etch by an oxygen-containing plasma [3-4]. Further study will include a HRTEM and EELS analysis of the outer surface of the ion implanted diamond to give evidence towards understanding the FIBHM masking mechanism.

The fabrication of diamond nano-photonic structures is one application where the FIBHM has demonstrated significant advantages as a new technique: Fig. 2 (A) shows a bright field Transmission Electron Microscope (TEM) image of a structure with dimensions suitable for nanophotonic waveguides. Sidewall and plateau RMS roughness values were measured in (B) to $<1 \mathrm{~nm}$ (to the resolution of the images), an improvement from 10-20nm using resist based techniques [4]. This improvement should significantly reduce light (surface roughness) scattering along the length of the waveguide. Fig. 2(C) and (D) demonstrate a novel photonic nanostructure expected to be realized by the improved patterned feature resolution of the FIBHM, known as single mode, antiresonant waveguides. The schematic in Fig. 2(C) shows modeling results of the optimum crosssectional dimensions for these structures. The first iteration in an attempt to create these waveguides is shown in the cross sectional TEM image in Fig. 2(D) The FIBHM layer is seen above the plateau of the waveguide structures masking the etch of the diamond to the appropriate lateral dimensions demonstrating a resolution sufficient to create the $\sim 25 \mathrm{~nm}$ gap required for this structure. 


\section{References}

[1] McKenzie W.R., Pethica J., and Cross G., Adv Mater (in submission) (2010).

[2] McKenzie, W.R, Pethica J., and Cross G., Microsc. Microanal., 15 (Suppl. 2) (2009) 328.

[3] Taniguchi, J. et al., Nanotechnology, 13 (5) (2002) 592.

[4] Hiscocks, M.P. et al., Diam Relat Mater, 17 (11) (2008) 1831.

[5] Authors would like to acknowledge CRANN at Trinity College Dublin where much of this experimental work originated, and also the ESTEEM program and Oxford Materials for access to their facilities and making the high resolution TEM component of this research possible.

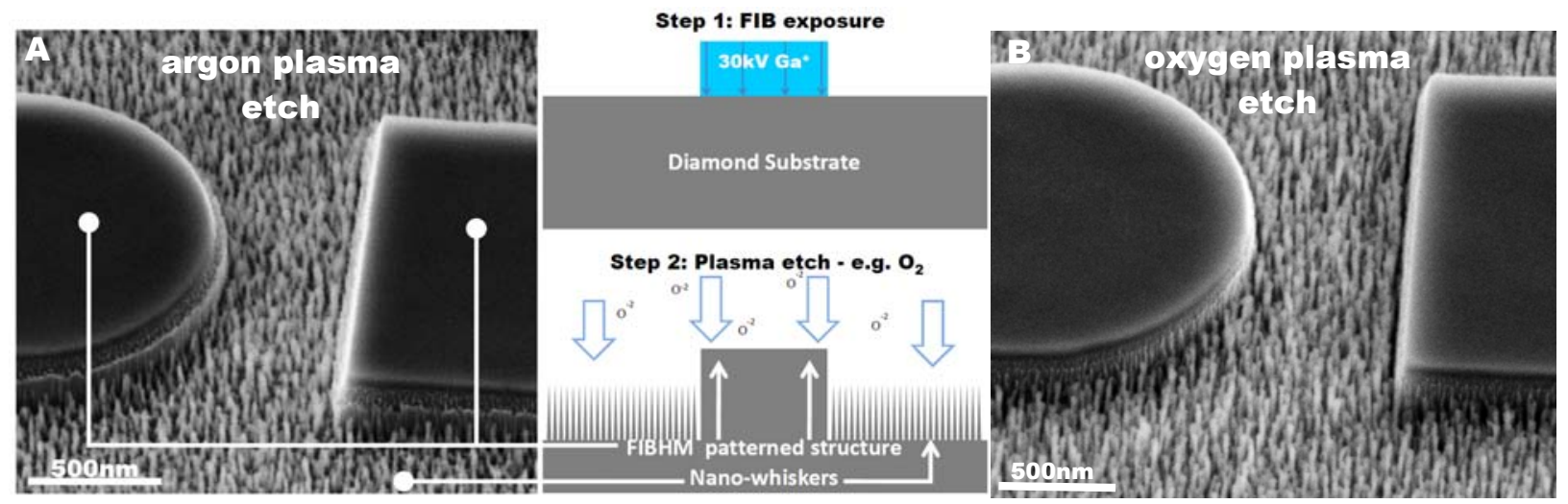

FIG 1. The Focused Ion Beam Hard Mask technique. Schematic - Step 1 of the FIBHM technique is a local exposure of the diamond surface with a $\mathrm{Ga}^{+}$FIB microscope. Step two is a plasma etch of the non-Ga exposed diamond leaving the patterned areas raised since they have been protected from the etch by the FIBHM. SEM images of structures created with the FIBHM technique using etches from (A) an argon plasma and (B) an oxygen plasma. The solid patterned structures and characteristics of the etched material appear very similar for both cases.
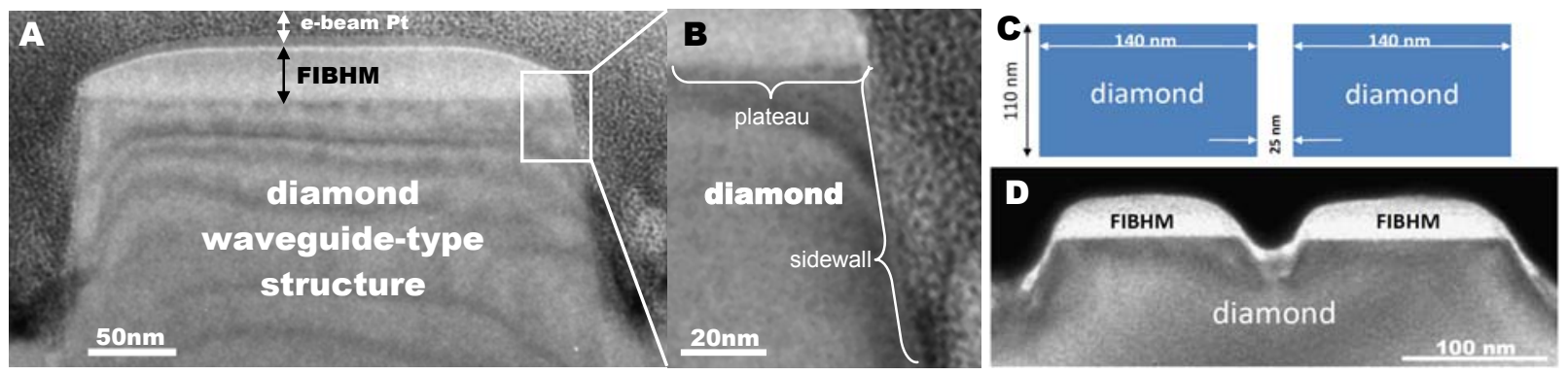

FIG 2. Preliminary experiments aimed at patterning a novel photonic structure. (A) bright field TEM image of a patterned structure created with the FIBHM. (B) high resolution TEM image of the inset in (A) highlighting the sidewall and plateau roughness of the diamond structure. (C) Schematic showing the optimum dimensions for single-mode anti-resonant waveguides as predicted by modeling using FIMMWAVE by Photon Design. (D) Bright-field cross-sectional TEM image of first attempts to fabricate these structures. 